\title{
Prevalencia y caracterización clínica del hipotiroidismo, en gestantes del Eje Cafetero (Colombia), 2014-2017*
}

\author{
Prevalence and clinical characterization of hypothyroidism in pregnant \\ women of the coffee region (Colombia), 2014-2017
}

\author{
Espitia $F^{1}$, Orozco L2.
}

${ }^{1}$ Médico especialista en Ginecología y Obstetricia, Universidad Militar Nueva Granada. Bogotá D.C., Colombia.

Máster en Sexología: Educación y asesoramiento sexual. Universidad de Alcalá de Henares. Madrid, España.

Uroginecología, FUCS - Hospital de San José. Unicamp, Brasil. Integrante del Servicio de Ginecología y Medicina Materno Fetal, Clínica La Sagrada Familia. Armenia, Quindío, Colombia.

${ }^{2}$ Médico Internista, Universidad El Bosque. Bogotá D.C., Colombia. Integrante del Servicio de Medicina Interna, Fundación Santa Fe de Bogotá. Bogotá D.C., Colombia.

Autor de correspondencia: Franklin José Espitia De La Hoz Correo electrónico: espitiafranklin@hotmail.com

Fecha de recepción: 07/06/2019

Fecha de aceptación: 30/07/2019

\section{Resumen}

Objetivo: conocer la prevalencia del hipotiroidismo en una población de mujeres gestantes del eje cafetero (Colombia).

Métodos: estudio observacional, descriptivo de corte transversal. Se incluyeron mujeres embarazadas con primer ingreso al control prenatal. A todas las gestantes se les realizó la prueba de perfil tiroideo con base en las recomendaciones de la Asociación Americana de Tiroides. Se incluyeron gestantes mayores de edad, con embarazo único, con historia clínica electrónica y sin enfermedad tiroidea preexistente que aceptaron participar en el estudio. Se excluyeron las gestantes con enfermedad neoplásica o hematológica, insuficiencia renal crónica o con anormalidades fetales congénitas. El estudio se hizo en tres clínicas del eje cafetero de nivel III, entre el 2014 y 2017. Se realizó un muestreo aleatorio sistemático consecutivo. Se midieron variables sociodemográficas, sintomatología de hipotiroidismo y antecedentes de salud sexual y reproductiva. Se realizó un análisis descriptivo de la información utilizando medidas de frecuencia absoluta y relativa.

Resultados: de un total de 579 mujeres seleccionadas, se analizaron finalmente $467(80,65 \%)$. La edad promedio fue de 24,85 (DE $\pm 5,3$ ) años. La prevalencia de hipotiroidismo en el grupo estudiado fue de $38,75 \%(n=181), 22,69 \%(n=106)$ con hipotiroidismo clínico, 15,99\% $(\mathrm{n}=75)$ con hipotiroidismo subclínico y el 12,41 \% $(n=58)$ presentó un trastorno tiroideo autoinmune.

Conclusión: las gestantes del eje cafetero presentan una alta prevalencia de hipotiroidismo, mayor a la reportada en otras zonas del mundo. Es necesario promocionar intervenciones oportunas para establecer un diagnóstico temprano y una terapéutica apropiada.

Palabras clave: hipotiroidismo, embarazo, factores de riesgo, diagnóstico, prevalencia, tiroides.

\section{Abstract}

Objective: To establish the prevalence of hypothyroidism in a population of pregnant women in the coffee region (Colombia).

Methods: Observational, descriptive cross-section study. Pregnant women of first admission to prenatal control were included. All pregnant women underwent the thyroid profile test based on the recommendations of the American Thyroid Association. We included pregnant women of legal age, with a single pregnancy, with electronic medical records, without pre-existing thyroid disease who agreed to participate in the study. Pregnant women with neoplastic or hematological disease, chronic renal insufficiency or with congenital fetal abnormalities were excluded. The study was carried out in three tertiary care centers of

*Trabajo ganador del primer lugar en presentación oral en el XV Congreso Colombiano de Endocrinología, Diabetes y Metabolismo. 
the coffee region between 2014 and 2017. A consecutive systematic random sampling was carried out. Socio-demographic variables, hypothyroidism symptoms and sexual and reproductive health antecedents were measured. A descriptive analysis of the information was made using absolute and relative frequency measurements.

Results: of a total of 579 women selected to participate, 467 (80.65\%) were finally analyzed. The average age was 24.85 (SD \pm 5.3) years. The prevalence of hypothyroidism in the studied group was $38.75 \%$ ( $n=181 / 479), 22.69 \%(n=106)$ with clinical hypothyroidism, $15.99 \%(n=75)$ subclinical hypothyroidism and $12.41 \%(n=58)$ presented an autoimmune thyroid disorder.

Conclusion: The pregnant women of the Coffee Region have a high prevalence of hypothyroidism, being higher than reported in other areas of the world. It is necessary to promote timely interventions to establish an early diagnosis and appropriate treatment.

Keywords: hypothyroidism, pregnancy, risk factors, diagnosis, prevalence, thyroid.

\section{Introducción}

Los trastornos de la tiroides no son excepcionales en las mujeres en edad reproductiva. La disfunción tiroidea en el embarazo puede influir de manera negativa en la salud del binomio madre-hijo y afectar la evolución del embarazo, así como el desarrollo físico y neurológico del neonato. Esta es la razón por la cual las guías clínicas sugieren realizar las pruebas de hormona estimulante de la tiroides tirotropina (TSH) y tiroxina libre (T4) a toda gestante con factores de riesgo ${ }^{(1)}$.

Durante el embarazo suceden una serie de interacciones materno-feto-placentarias en las que ocurre un incremento de las concentraciones de la proteína transportadora de hormonas tiroideas (TBG). Esto se debe al incremento de los estrógenos, lo que redunda en un aumento de las hormonas tiroideas ${ }^{(2)}$. La gonadotropina coriónica humana (hCG), al presentar homología con la hormona estimulante de la tiroides, actúa estimulando la tiroides y disponiendo la disminución de la TSH materna en el primer trimestre, con una restauración subsiguiente ${ }^{(3)}$. Es por esto por lo que la mayoría de los investigadores coinciden al aceptar como valor máximo de TSH en el primer trimestre un rango hasta $2,5 \mathrm{mIU} / \mathrm{L}^{(1,4)}$. Los valores normales utilizados para la TSH son los recomendados por la American Endocrine Society (AES) y la American Thyroid Association (ATA) (Tabla 1) ${ }^{(1,5)}$.

El hipotiroidismo es una enfermedad que se caracteriza por la disminución en la síntesis y secreción de las hormonas tiroideas o por un defecto en la actividad del receptor de las hormonas tiroideas. Esto desata un estado hipometabólico generalizado y desencadena repercusiones negativas significativas en todo el organismo ${ }^{(6)}$.

La prevalencia del hipotiroidismo gestacional oscila entre el $2 \%$ y $5 \%^{(7,8)}$ : 0,3 \%-1 \% para el hipotiroidismo clínico y $3 \%-5 \%$ para el hipotiroidismo subclínico ${ }^{(9,10)}$. No obstante, la prevalencia varía de acuerdo con los criterios diagnósticos ${ }^{(7,}$ 11), el trimestre del embarazo ${ }^{(12)}$, el estado nutricional de yodo materno $^{(13)}$, así como de la edad y la raza.

El diagnóstico clínico se basa en una serie de manifestaciones clínicas inespecíficas (Tabla 2) ${ }^{(14)}$. Los valores diagnósticos de la TSH se han definido por trimestres, pero, en ausencia de rangos propios de referencia, se recomienda aceptar los descritos en la Tabla 1 (valores inferiores a los de la población general) ${ }^{(15,16)}$.

La TSH se debe cuantificar al comienzo del embarazo, antes de la 9. ${ }^{a}$ semana. Si se encuentra aumentada se procede a la medición de la tiroxina libre (T4L) y de los anticuerpos anti-tiroperoxidasa (TPOAb), para definir si se trata de un hipotiroidismo clínico o subclínico ${ }^{(1,3,5)}$. Se sugiere seguir el esquema descrito en la Figura 1.

El hipotiroidismo clínico o subclínico en la gestante suelen tener efectos adversos en el feto; por lo tanto, se deben tratar sin retraso. La levotiroxina oral es la elección junto con suplementos adecuados de yodo. La meta es alcanzar valores de TSH dentro del rango específico para cada trimestre del embarazo, según la Tabla $\mathbf{1}^{(1,3,5,17)}$.

El objetivo de este estudio fue determinar la prevalencia del hipotiroidismo clínico y subclínico en una población de mujeres embarazadas que acudieron a control prenatal en tres clínicas del eje cafetero y caracterizar las manifestaciones clínicas, ya que el hipotiroidismo gestacional se asocia con pobres resultados obstétricos y retraso mental en la descendencia sobreviviente.

Tabla 1. Valores de referencia de TSH maternos por trimestre del embarazo

Trimestre de gestación

Primero

Segundo

Tercero
TSH (mUI/L)

$0,1-2,5$

$0,2-3,0$

$0,3-3,0$ 
Tabla 2. Manifestaciones clínicas del hipotiroidismo

\begin{tabular}{|c|c|c|}
\hline & Síntomas & Signos \\
\hline Generales & $\begin{array}{l}\text { Intolerancia al calor } \\
\text { Astenia } \\
\text { Aumento de peso }\end{array}$ & Hipotermia \\
\hline Neurológicas & $\begin{array}{l}\text { Pérdida de la memoria } \\
\text { Cambios en la personalidad }\end{array}$ & $\begin{array}{l}\text { Somnolencia } \\
\text { Bradilalia } \\
\text { Bradipsiquia } \\
\text { Psicosis hipotiroidea } \\
\text { Reducción de la audición y el sentido del gusto } \\
\text { Ataxia y retraso de la fase de relajación de los reflejos } \\
\text { osteotendinosos } \\
\text { Calambres }\end{array}$ \\
\hline Neuromusculares & $\begin{array}{l}\text { Debilidad } \\
\text { Dolor articular }\end{array}$ & $\begin{array}{l}\text { Rigidez articular } \\
\text { Síndrome del túnel carpiano }\end{array}$ \\
\hline Gastrointestinales & $\begin{array}{l}\text { Náuseas } \\
\text { Estreñimiento }\end{array}$ & $\begin{array}{l}\text { Macroglosia } \\
\text { Ascitis }\end{array}$ \\
\hline Cardiorrespiratorias & Disminución de la tolerancia al ejercicio físico & $\begin{array}{l}\text { Voz grave y bradilalia } \\
\text { Bradicardia } \\
\text { Hipertensión leve/moderada } \\
\text { Derrame pericárdico } \\
\text { Derrame pleural }\end{array}$ \\
\hline $\begin{array}{l}\text { Esfera gonadal y ge- } \\
\text { nital }\end{array}$ & $\begin{array}{l}\text { Reducción de la libido } \\
\text { Disminución de la fertilidad } \\
\text { Alteraciones menstruales }\end{array}$ & \\
\hline Piel y faneras & $\begin{array}{l}\text { Piel áspera y fría } \\
\text { Edema facial } \\
\text { Caída del vello } \\
\text { Caída del cabello }\end{array}$ & $\begin{array}{l}\text { Coloración pálida o amarilla } \\
\text { Cabello áspero y quebradizo } \\
\text { Reducción del pelo del tercio distal de las cejas } \\
\text { Uñas estriadas y quebradizas } \\
\text { Axilas secas } \\
\text { Edema prioritario } \\
\text { Edema sin fóvea en cara, dorso de las manos y tobillos }\end{array}$ \\
\hline
\end{tabular}


Figura 1. Abordaje diagnóstico y terapéutico de la gestante con disfunción tiroidea.

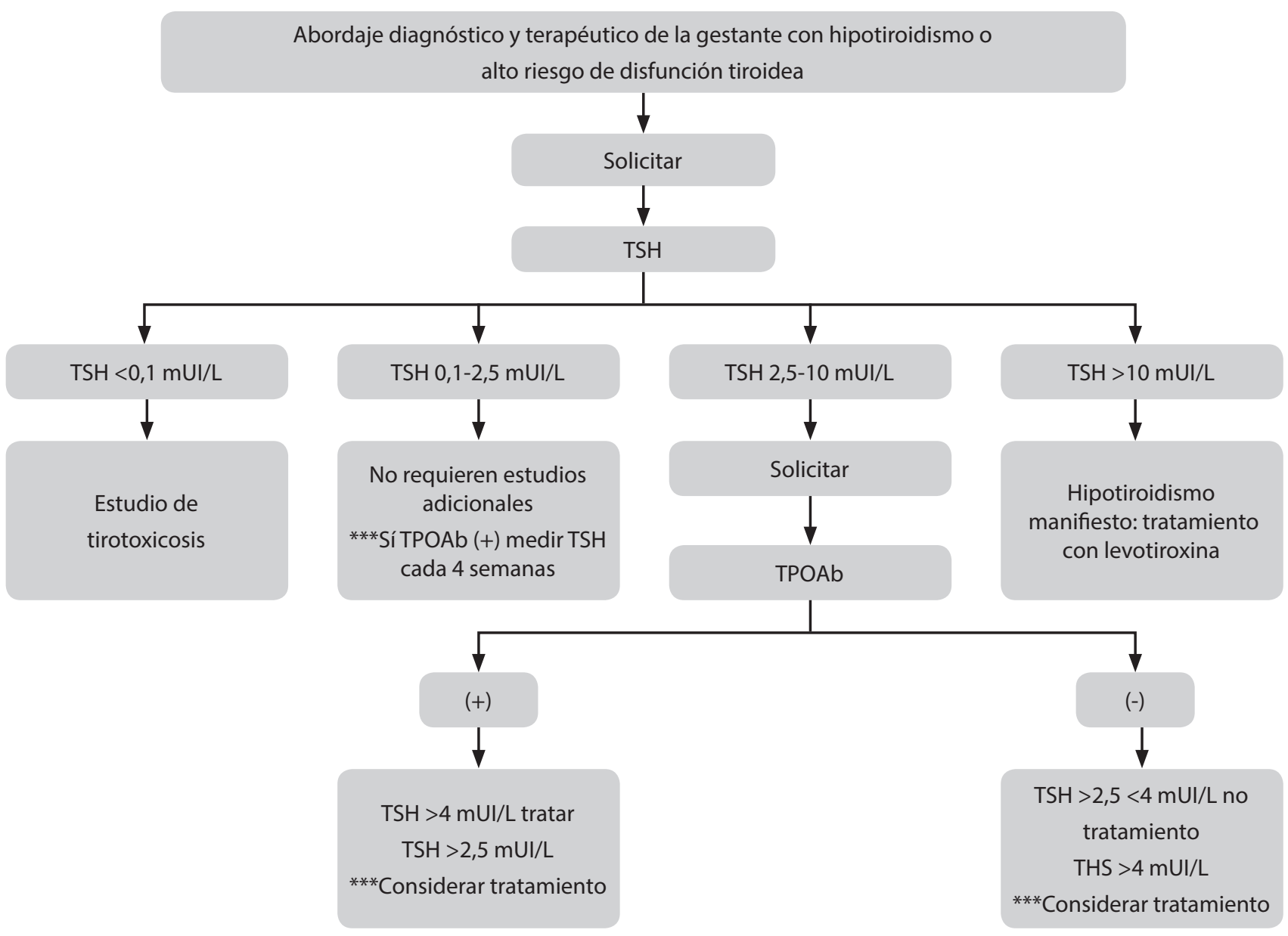

\section{Materiales y métodos}

\section{Diseño y población}

Estudio observacional, descriptivo de corte transversal, realizado del 1 de agosto de 2014 al 31 de julio de 2017, en la consulta externa de tres clínicas de las ciudades de Manizales, Pereira y Armenia en el eje cafetero (ubicado en la región central de Colombia). Estas clínicas son de atención de alta complejidad, de carácter universitario, que atienden a personas pertenecientes a los regímenes de aseguramiento contributivo y subsidiado por el Estado en el sistema de seguridad social en Colombia.

Se incluyeron gestantes mayores de edad, con embarazo único, con historia clínica electrónica y sin enfermedad tiroidea preexistente que aceptaron participar en el estudio. Se excluyeron las gestantes con enfermedad neoplásica o hematológica, insuficiencia renal crónica, con anormalidades fetales congénitas y las que no consintieron en participar en el estudio.

La muestra se obtuvo mediante muestreo aleatorio sistemático consecutivo y la integraron 579 gestantes; sin em- bargo, solo 467 (80,65 \%) mujeres fueron incluidas. Se hizo el cálculo del tamaño de la muestra tomando como valor de referencia una prevalencia esperada del $20 \%$, un nivel de confianza del 95 \% y una precisión del 0,05. El tamaño óptimo de la muestra resultante fue de 452 casos.

\section{Procedimiento}

Se seleccionaron aleatoriamente las pacientes de la población de mujeres adscritas al programa de atención prenatal de cada institución participante, entre las semanas 6 y 39 de gestación y con edades comprendidas entre 18 y 42 años. La recolección de la información estuvo a cargo de las enfermeras entrenadas en el proceso de captación de pacientes y diligenciamiento de formularios, quienes conforman el equipo de investigación. Estas encuestaron a las gestantes antes de ingresar a la consulta con el médico, teniendo en cuenta los criterios de inclusión y exclusión. A las que cumplían con los criterios de selección y aceptaron participar en el estudio se les explicaron los objetivos de la investigación y se les solicitó 
diligenciar el consentimiento informado, garantizándose así la confidencialidad en el manejo de la información.

A una de cada dos gestantes que asistieron al control se les tomó la muestra de sangre para la realización de la tirotropina (TSH). Una vez se tomaron las muestras de laboratorio, se les aplicó un cuestionario de autorreporte en el que se registraron los antecedentes biológicos sociales y demográficos (edad, estado civil, raza, nivel de estudios, ocupación, antecedentes personales y familiares, medicamentos, ingesta de alcohol y tabaquismo), sintomatología, factores de riesgo y nivel de actividad física. Además, se tomaron medidas antropométricas (peso, talla e índice de masa corporal) y antecedentes ginecoobstétricos (última menstruación, edad gestacional y paridad). Finalmente, se les realizó un examen físico general y del cuello para determinar la presencia de bocio.

En ayunas, a cada gestante se le extrajo $5 \mathrm{~mL}$ de sangre de una vena braquial, entre las 8:00 y las 10:00 de la mañana, a lo largo del período de estudio. Las muestras se centrifugaron y analizaron el mismo día de la extracción. Se midió la TSH, la cual se procesó de inmediato en el laboratorio de cada uno de los centros de atención, mediante quimioluminiscencia amplificada, con un equipo Immulite 2000 Siemens. Los resultados se entregaron diariamente a las gestantes.

Cuando las embarazadas del primer trimestre presentaron un nivel de TSH $>2,5 \mathrm{mUI} / \mathrm{L}$ o las del segundo o tercer trimestre mostraron un nivel de TSH >3,0 mUI/L, se les citaba nuevamente para realizar un análisis de confirmación de la TSH entre la primera y segunda semana después. En esta ocasión, adicional a la TSH, se realizó la medición cuantitativa de la tiroxina libre (T4L) en suero (aceptand o como niveles normales valores entre $0,80-1,80 \mathrm{ng} / \mathrm{dL}$ en el primer, el segundo y el tercer trimestre) y los anticuerpos anti-tiroperoxidasa (TPOAb) (se consideraron niveles elevados para los TPOAb, de acuerdo con el punto de corte sugerido por el fabricante del equipo utilizado [Immulite]: $35 \mathrm{UI} / \mathrm{mL}$ ). La información referente a los resultados de laboratorio y los hallazgos del examen físico se registró en la historia clínica y en un formato especial en Excel diseñado por los investigadores.

\section{Variables medidas}

Se tuvieron en cuenta las siguientes variables: edad, raza, ocupación, peso, talla, IMC, variables sociodemográficas, antecedentes de salud sexual y reproductiva (edad de inicio de la actividad sexual, edad del primer embarazo, paridad y número de embarazos) y sintomatología de hipotiroidismo (Tabla 2). Se consideró hipotiroidismo cuando las concentraciones de TSH eran mayores de 2,5 UI/mL en el primer trimestre y mayores de $3 \mathrm{UI} / \mathrm{mL}$ en el segundo y tercer trimestre, según las guías de manejo de la Asociación Americana de Tiroides ${ }^{(18)}$. También se clasificó en hipotiroidismo clínico (TSH elevada y T4L baja) o hipotiroidismo subclínico (TSH elevada, menor de 10 mUI/L y T4L normal).

\section{Análisis estadístico}

El análisis se realizó en los programas estadísticos SPSS versión 17.0 (SPSS Inc. Chicago, Illinois) y STATA SE 10.1. Se calculó la prevalencia y se expresó en porcentaje. Las variables numéricas se expresaron como media, con su desviación estándar y rangos; y se extrajo la mediana. Las variables categóricas se expresaron como frecuencias absolutas y porcentajes.

\section{Aspectos bioéticos}

El estudio cumplió con las consideraciones éticas para la investigación en seres humanos, y tanto el protocolo de investigación como el consentimiento informado fueron avalados por los Comités de Ética de las clínicas participantes. También se garantizó la confidencialidad del manejo de la información. Las gestantes con resultados anormales de las pruebas diagnósticas se incluyeron en el protocolo de atención de gestantes de alto riesgo en cada una de las clínicas participantes, donde fueron tratadas por personal médico especializado.

\section{Resultados}

Se reclutó un total de 579 gestantes candidatas a participar en la investigación. Se detectaron $9(1,55 \%)$ con leucemia aguda, $6(1,03 \%)$ con linfoma y $68(11,74 \%)$ con trombocitopenia gestacional. De las 496 mujeres restantes, 29 (5,84\%) no se realizaron la T4L ni los Ac-TPO. De esta manera, un total de 467 (80,65 \%) gestantes participaron en el estudio.

La edad media de las participantes fue 24,85 [desviación estándar (DE) \pm 5,3] años, rango entre 18 y 42 años, la mayoría amas de casa, en unión libre, de estrato medio, residentes del área urbana, hispánicas, multigestantes y del régimen contributivo. El IMC promedio fue 26,3 (DE \pm 5,8), el 38,6 \% presentó IMC de $\geq 25$. La Tabla 3 muestra las características sociodemográficas de las gestantes.

Los antecedentes de salud sexual y reproductiva mostraron una mediana de 2 hijos en la paridad (rango entre 0 y 7 hijos). En relación con el número de embarazos, se encontró una mediana de 4 gestaciones (rango entre 0 y 11 embarazos). Un total de 357 $(76,44 \%)$ gestantes reportaron una mediana de 1 aborto (rango entre 1 y 3 abortos), y 314 (67,23 \%) de los embarazos actuales fueron no planeados. En cuanto a la vía del parto, la cesárea reportó una mediana de 2 , con un rango entre 1 y 6 . Respecto a la edad del inicio de la actividad sexual, el promedio fue de 16,5 $(\mathrm{DE} \pm 2,4)$ años. En relación con el primer parto, la edad promedio fue de 16,8 (DE $\pm 2,7$ ) años, con una mediana de 17 años.

Al momento de la toma del examen, el 46,89 \% ( $n=219)$ de las gestantes cursaba el primer trimestre del embarazo, $34,47 \%(n=161)$ el segundo trimestre y $18,62 \%(n=87)$ el tercer trimestre. La mediana de la edad gestacional al momento de la realización del examen fue de 17 semanas, con rango entre 6 y 39 semanas. Un total de 113 de las 467 gestantes $(24,19 \%)$ iniciaron el control prenatal de manera tardía. 
Tabla 3. Características sociodemográficas y en salud sexual y reproductiva de la población de gestantes con hipotiroidismo en el Eje Cafetero (Colombia), 2014-2017

\begin{tabular}{|c|c|}
\hline Variable y categorías & $n=467$ \\
\hline Edad (años) \pm DE & $24,85 \pm 5,3$ \\
\hline Talla (centímetros) $\pm \mathrm{DE}$ & $159,74 \pm 6,52$ \\
\hline $\mathrm{IMC} \pm \mathrm{DE}$ & $26,3 \pm 5,8$ \\
\hline \multicolumn{2}{|l|}{ Raza } \\
\hline Hispánicas & $266(56,95 \%)$ \\
\hline Indígenas & $89(19,05 \%)$ \\
\hline Afrocolombianas & $112(23,98 \%)$ \\
\hline \multicolumn{2}{|l|}{ Estado civil } \\
\hline Casada & $131(28,05 \%)$ \\
\hline Unión libre & $224(47,96 \%)$ \\
\hline Soltera & $81(17,34 \%)$ \\
\hline Separada-divorciada & $20(4,28 \%)$ \\
\hline Viuda & $11(2,35 \%)$ \\
\hline \multicolumn{2}{|l|}{ Estrato socioeconómico } \\
\hline Alto & $69(14,77 \%)$ \\
\hline Medio & $223(47,75 \%)$ \\
\hline Bajo & $175(37,47 \%)$ \\
\hline \multicolumn{2}{|l|}{ Ocupación } \\
\hline Amas de casa & $322(68,95 \%)$ \\
\hline Empleadas & $145(31,04 \%)$ \\
\hline \multicolumn{2}{|l|}{ Aseguramiento } \\
\hline Contributivo & $337(72,16 \%)$ \\
\hline Subsidiado & $130(27,83 \%)$ \\
\hline \multicolumn{2}{|l|}{ Gravidez } \\
\hline Primigestante & $88(18,84 \%)$ \\
\hline Multigestante & $379(81,15 \%)$ \\
\hline \multicolumn{2}{|l|}{ Origen } \\
\hline Urbano & $268(57,38 \%)$ \\
\hline Rural & $199(42,61 \%)$ \\
\hline
\end{tabular}

En la población total de las 467 gestantes, el promedio de la TSH en el primer trimestre fue de 2,02 mUI/L (DE $\pm 0,84$ ); este subió a 2,39 $\mathrm{mUI} / \mathrm{L}(\mathrm{DE} \pm 0,73$ ) en el segundo trimestre y a $2,65 \mathrm{mUI} / \mathrm{L}(\mathrm{DE} \pm 0,92)$ en el tercer trimestre. La puntua- ción de la TSH en la población total de las 467 gestantes fue de $2,03 \mathrm{mUI} / \mathrm{L}(\mathrm{DE} \pm 0,86$ ). El valor máximo fue de $16,35 \mathrm{mUI} / \mathrm{L}$ y el mínimo 0,76 mUI/L. El promedio de la T4L fue 1,07 ng/ $\mathrm{dL}(\mathrm{DE} \pm 0,89$ ) (rango entre 0,56 y $3,07 \mathrm{ng} / \mathrm{dL}$ ). En el grupo de embarazadas con hipotiroidismo, la media de la puntuación de la TSH fue de $4,79 \mathrm{mUI} / \mathrm{L}$ ( $\mathrm{DE} \pm 1,49$ ), donde el valor máximo fue de 16,35 mUI/L (DE $\pm 1,49$ ) y el mínimo de 3,36 mUI/L. La media de la T4L fue $0,72 \mathrm{ng} / \mathrm{dL}(\mathrm{DE} \pm 0,23)$ con un rango entre 0,26 y $0,77 \mathrm{ng} / \mathrm{dL}$.

El 38,75 \% (181/467) arrojó una TSH >2,5 mUI/L; el 22,69 \% (106/181) presentó una T4L de 0,53 ng/dL (DE \pm 0,37) y 12,41 \% (58/181) reportó un valor elevado de Ac-TPO de $38,14 \mathrm{UI} / \mathrm{mL}(\mathrm{DE} \pm 8,9)$, que se consideraron como casos positivos de trastorno tiroideo autoinmune durante el embarazo. Las gestantes con reportes aumentados en los Ac-TPO mostraron valores promedios de TSH elevados $(14,08 \mathrm{mUI} / \mathrm{L}[\mathrm{DE} \pm 3,85])$.

La prevalencia de hipotiroidismo en este grupo de gestantes fue de 38,75 \% ( $n=181 / 479) ; 22,69 \%(n=106)$ con hipotiroidismo clínico y $15,99 \%(n=75)$ con hipotiroidismo subclínico. El 12,41 \% (n = 58) presentó niveles elevados en los Ac-TPO.

La prevalencia de la sintomatología clínica en el grupo estudiado fue de $36,83 \%(n=172 / 467)$. El síntoma que se presentó con mayor frecuencia fue el estreñimiento con 172 casos (36,83\%), seguido por las parestesias con 158 casos $(33,83 \%)$ y, en tercer lugar, la piel fría con 103 casos $(22,05 \%)$; el menos frecuente fue la ronquera con 74 casos $(15,84 \%)$. Se encontró que, de las 172 gestantes con sintomatología, el 21,51 \% presentaba un síntoma, el 44,76 \% dos y el $35,46 \%$ tres o más síntomas, con una mediana de 5 síntomas por gestante (rango entre 1 y 10). En la Tabla 4 se describe la prevalencia detallada de cada síntoma en la población de gestantes con hipotiroidismo.

Tabla 4. Prevalencia síntomas de hipotiroidismo en una población de gestantes del eje cafetero

\begin{tabular}{l|c|c}
\multicolumn{1}{c|}{ Síntomas } & $\mathbf{n}$ & $\%$ \\
\hline Aumento de peso & 94 & 20,12 \\
\hline Disminución de la sudoración & 91 & 19,48 \\
\hline Edema periorbitario & 125 & 26,76 \\
\hline Estreñimiento & 172 & 36,83 \\
\hline Parestesias & 158 & 33,83 \\
\hline Piel fría & 103 & 22,05 \\
\hline Piel gruesa & 83 & 17,77 \\
\hline Piel seca & 77 & 16,48 \\
\hline Ronquera & 74 & 15,84 \\
\hline
\end{tabular}


De las 172 gestantes que presentaron sintomatología clínica de hipotiroidismo, el 61,62 \% (n=106) se diagnosticó con hipotiroidismo clínico; y 38,37 \% ( $\mathrm{n}=66)$, con hipotiroidismo subclínico. De estos casos, el 23,83 \% (n= 41) se consideraron como positivos de trastorno tiroideo autoinmune (hipotiroidismo clínico 15,69 \% [n=27/172], e hipotiroidismo subclínico $8,13 \%[n=14 / 172])$.

\section{Discusión}

En esta investigación se encontró una prevalencia de hipotiroidismo de 38,75 \%. Respecto al resultado de la TSH, se evidenció una prevalencia de 22,69 \% de hipotiroidismo clínico y $15,99 \%$ de hipotiroidismo subclínico; de estos casos, el $12,41 \%$ presentó un trastorno tiroideo autoinmune, en donde la prevalencia de manifestaciones clínicas fue de 36,83 \%. El estreñimiento $(36,83 \%)$ fue el síntoma que se presentó con mayor frecuencia, seguido por las parestesias $(33,83 \%)$ y, en tercer lugar, la piel fría (22,05 \%). Asimismo, se observó que es inusual la presencia de un solo síntoma $(21,51 \%)$.

$\mathrm{Al}$ contrastar la prevalencia del hipotiroidismo gestacional encontrada en este estudio, se observan cifras superiores a las reportadas por autores internacionales. Se evidencia que dicha prevalencia fue inferior en Maine (Estados Unidos), pues Klein y colaboradores ${ }^{(19)}$ reportaron un $0,3 \%$ en mujeres de 15 a 18 semanas de gestación. En la India, Nambiar y colaboradores $^{(20)}$ reportaron un 4,8 \% en embarazadas en el primer trimestre. Estos datos son inferiores a lo documentado por Fukushi y su equipo de investigadores ${ }^{(21)}$ en gestantes japonesas $(0,14 \%)$ o a lo informado por Wang y colaboradores ${ }^{(22)}$ en China $(10,9 \%$ en el primer trimestre).

Lo anterior se podría explicar por la edad gestacional en la que se hizo la cuantificación de la TSH, el mayor tamaño de la muestra, así como por los criterios de inclusión de los estudios y las diferencias en aspectos raciales. No pudimos contrastar nuestros resultados con reportes del país, ya que no encontramos estudios similares en Colombia.

El nivel promedio de la TSH fue 4,79 mUI/L (DE $\pm 1,49$ ) en las gestantes con hipotiroidismo (rango entre 3,36 y 16,35 mUI/L); este promedio es mucho mayor a la recomendación aceptada como límite superior normal: 2,5 mUI/L ${ }^{(1)}$. Además, se detectó un elevado valor de Ac-TPO (12,41\%), pero inferior a los resultados de Bocos-Terraz y colaboradores ${ }^{(23)}$, que reportaron 14,77 \% en España. Esto se asocia con la presencia de enfermedades autoinmunes de la tiroides, lo que explicaría las concentraciones elevadas de TSH en la población total.

La prevalencia de hipotiroidismo subclínico en este estudio reporta cifras superiores en comparación con los hallazgos de otras investigaciones (1\% a $2 \%)^{(24,25)}$. De acuerdo con lo reportado por Casey y colaboradores ${ }^{(26)}$, esto hace previsible el incremento de las probabilidades de acontecimientos adversos. El American College of Obstetricians and Ginecologyst ${ }^{(27)}$ recomienda implementar el tratamiento de manera justificada, en especial si se acompaña de la presencia de anticuerpos antitiroideos, ya que estaría asociada con procesos autoinmunes.

Respecto a las manifestaciones clínicas, nuestros datos no pudieron ser contrastados con otros estudios, ya que no encontramos ningún trabajo que caracterizara la prevalencia de los signos y síntomas en las mujeres embarazadas. Este estudio guía a enfatizar la exploración clínica exhaustiva en las gestantes con la sintomatología descrita (Tabla 2), puesto que ayuda en la orientación del diagnóstico de hipotiroidismo, ya que de las 181 gestantes a las cuales se les detectó hipotiroidismo, el 95,02 \% ( $\mathrm{n}=172 / 181$ ) presentó sintomatología clínica.

Las gestantes con hipotiroidismo (clínico o subclínico) se asocian con mayor riesgo de abortos, anemia, desprendimiento prematuro de placenta, hemorragia posparto, diabetes gestacional, hipertensión gestacional, prematuridad, bajo peso al nacer, muerte fetal, distrés respiratorio, daño neurológico, disminución del coeficiente de inteligencia, etc. $\left({ }^{28-30}\right)$. Al observar este estudio, se estima que una de cada tres gestantes, del eje cafetero, presenta hipotiroidismo; por lo tanto, consideramos la tamización, no como un tema de controversia, sino como de implementación obligatoria.

Se destacan como debilidades de este estudio la falta de seguimiento de las pacientes hasta el final del embarazo y el puerperio, por lo que no se pudo evaluar el comportamiento de la terapia. De igual modo, no haber ampliado el estudio a las demás regiones del país obliga a excluir a muchas gestantes, situación que podría introducir un sesgo en la prevalencia a nivel de las mujeres embarazadas en Colombia. Entre las fortalezas de esta investigación señalamos que es el primer estudio de estas características realizado en el país; lo otro es que, debido a lo significativo de la muestra, se tiene una adecuada información sobre una población representativa de gestantes colombianas, con una amplia variedad de edades, razas y estratos socioeconómicos.

Los resultados de esta investigación señalan un importante desafío para próximos estudios sobre la prevalencia del hipotiroidismo en gestantes colombianas. Sería primordial vincular una mayor participación de mujeres embarazadas en las distantes ciudades del país. Amparados en los presentes resultados, invitamos al personal de la salud a solicitar la TSH de rutina en el primer control prenatal (idealmente antes de la 9. ${ }^{\text {a }}$ semana).

\section{Conclusiones}

En las gestantes del eje cafetero existe una elevada prevalencia de hipotiroidismo, principalmente de hipotiroidismo clínico; dicha prevalencia es mayor a la reportada en otras zonas del mundo. Se advierte la necesidad de estudios poblacionales para sopesar estas estimaciones en la comunidad general de embarazadas colombianas. 


\section{Agradecimientos}

Nuestro sincero agradecimiento al personal de enfermería que nos apoya en estos proyectos; a la doctora Ana María Londoño Zapata, por haber colaborado en la presentación de este estudio en el 15 Congreso Colombiano de Endocrinología, Diabetes y Metabolismo, donde obtuvo el primer lugar en presentación oral; a la doctora Diana Marcela Echeverri Muñoz, por ayudar en la diagramación; así como a mis apreciados maestros, los doctores John Jairo Duque Ossman y Oscar Elías
Zuluaga Cortés, por enriquecerme con sus recomendaciones y sugerencias en la elaboración de esta investigación.

\section{Financiación}

El proyecto fue financiado con recursos propios de los autores.

\section{Conflictos de interés}

Los autores declaran no tener ningún conflicto de interés.

\section{Referencias}

1. Stagnaro-Green A, Abalovich M, Alexander E, Azizi F, Mestman J, Negro R et al. Guidelines of the American Thyroid Association for the diagnosis and management of thyroid disease during pregnancy and postpartum. Thyroid Off J Am Thyroid Assoc. 2011;21(10):1081-125.

2. Negro R, Mestman JH. Thyroid disease in pregnancy. Best Pract Res Clin Endocrinol Metab. 2011;25(6):927-43

3. Lazarus J, Brown RS, Daumerie C, Hubalewska-Dydejczyk A, Negro R, Vaidya B. 2014 European thyroid association guidelines for the management of subclinical hypothyroidism in pregnancy and in children. Eur Thyroid J. junio de 2014;3(2):76-94.

4. Negro R, Schwartz A, Gismondi R, Tinelli A, Mangieri T, Stagnaro-Green A Increased pregnancy loss rate in thyroid antibody negative women with TSH levels between 2.5 and 5.0 in the first trimester of pregnancy. J Clin Endocrinol Metab. 2010;95(9):E44-48.

5. De Groot L, Abalovich M, Alexander EK, Amino N, Barbour L, Cobin RH, et al. Management of thyroid dysfunction during pregnancy and postpartum: an Endocrine Society clinical practice guideline. J Clin Endocrinol Metab. 2012;97(8):2543-65.

6. Rashid M, Rashid MH. Obstetric Management of Thyroid Disease: Obstet Gynecol Surv. 2007;62(10):680-8.

7. Casey BM, Dashe JS, Spong CY, McIntire DD, Leveno KJ, Cunningham GF. Perinatal Significance of Isolated Maternal Hypothyroxinemia Identified in the First Half of Pregnancy: Obstet Gynecol. 2007;109(5):1129-35.

8. Cleary-Goldman J, Malone FD, Lambert-Messerlian G, Sullivan L, Canick J, Porter TF, et al. Maternal thyroid hypofunction and pregnancy outcome. Obstet Gynecol. 2008:112(1):85-92.

9. Lazarus JH, Bestwick JP, Channon S, Paradice R, Maina A, Rees R, et al. Antenatal Thyroid Screening and Childhood Cognitive Function. N Engl J Med. 2012;366(6):493-501.

10. Potlukova E, Potluka O, Jiskra J, Limanova Z, Telicka Z, Bartakova J, et al. Is age a risk factor for hypothyroidism in pregnancy? An analysis of 5223 pregnant women. J Clin Endocrinol Metab. 2012;97(6):1945-52.

11. Allan WC, Haddow JE, Palomaki GE, Williams JR, Mitchell ML, Hermos RJ, et al. Maternal thyroid deficiency and pregnancy complications: implications for population screening. J Med Screen. 2000;7(3):127-30.

12. Blatt AJ, Nakamoto JM, Kaufman HW. National status of testing for hypothyroidism during pregnancy and postpartum. J Clin Endocrinol Metab. 2012;97(3):777-84.

13. Glinoer D. Pregnancy and Iodine. Thyroid. 2001;11(5):471-81.

14. Fitzpatrick DL, Russell MA. Diagnosis and management of thyroid disease in pregnancy. Obstet Gynecol Clin North Am. 2010;37(2):173-93.

15. Granfors M, Åkerud H, Berglund A, Skogö J, Sundström-Poromaa I, Wikström A-K. Thyroid testing and management of hypothyroidism during pregnancy: a population-based study. J Clin Endocrinol Metab. 2013;98(7):2687-92.

16. Amouzegar A, Ainy E, Khazan M, Mehran L, Hedayati M, Azizi F. Local versus international recommended TSH references in the assessment of thyroid function during pregnancy. Horm Metab Res Horm Stoffwechselforschung Horm Metab. 2014;46(3):206-10.

17. Pearce SHS, Brabant G, Duntas LH, Monzani F, Peeters RP, Razvi S, et al 2013 ETA Guideline: Management of Subclinical Hypothyroidism. Eur Thyroid J. 2013;2(4):215-28.

18. Alexander EK, Pearce EN, Brent GA, Brown RS, Chen H, Dosiou C, et al. 2017 Guidelines of the American Thyroid Association for the Diagnosis and Management of Thyroid Disease During Pregnancy and the Postpartum. Thyroid Off J Am Thyroid Assoc. 2017;27(3):315-89.

19. Klein RZ, Haddow JE, Faix JD, Brown RS, Hermos RJ, Pulkkinen A, et al Prevalence of thyroid deficiency in pregnant women. Clin Endocrinol (Oxf). 1991;35(1):41-6.

20. Nambiar V, Jagtap VS, Sarathi V, Lila AR, Kamalanathan S, Bandgar TR et al. Prevalence and Impact of Thyroid Disorders on Maternal Outcome in Asian-Indian Pregnant Women. J Thyroid Res. 2011;2011:1-6.

21. Fukushi M, Honma K, Fujita K. Maternal thyroid deficiency during pregnancy and subsequent neuropsychological development of the child. $\mathrm{N}$ Engl J Med. 1999;341(26):2016; author reply 2017.

22. Wang W, Teng W, Shan Z, Wang S, Li J, Zhu L, et al. The prevalence of thyroid disorders during early pregnancy in China: the benefits of universal screening in the first trimester of pregnancy. Eur J Endocrinol. 2011;164(2):263-8

23. Bocos-Terraz JP, Izquierdo-Álvarez S, Bancalero-Flores JL, Álvarez-Lahuerta R, Aznar-Sauca A, Real-López E, et al. Thyroid hormones according to gestational age in pregnant Spanish women. BMC Res Notes. 2009;2:237.

24. Abalovich M, Gutierrez S, Alcaraz G, Maccallini G, Garcia A, Levalle 0. Overt and Subclinical Hypothyroidism Complicating Pregnancy. Thyroid. 2002;12(1):63-8

25. Casey BM, Leveno KJ. Thyroid disease in pregnancy. Obstet Gynecol. 2006;108(5):1283-92.

26. Casey BM, Dashe JS, Wells CE, McIntire DD, Byrd W, Leveno KJ, et al Subclinical hypothyroidism and pregnancy outcomes. Obstet Gynecol. 2005;105(2):239-45.

27. Alexander EK, Marqusee E, Lawrence J, Jarolim P, Fischer GA, Larsen PR. Timing and magnitude of increases in levothyroxine requirements during pregnancy in women with hypothyroidism. N Engl J Med. 2004;351(3):241-9.

28. Henrichs J, Bongers-Schokking JJ, Schenk JJ, Ghassabian A, Schmidt HG, Visser TJ, et al. Maternal thyroid function during early pregnancy and cognitive functioning in early childhood: the generation R study. J Clin Endocrinol Metab. 2010;95(9):4227-34.

29. Morreale de Escobar G, Obregon MJ, Escobar del Rey F. Role of thyroid hor mone during early brain development. Eur J Endocrinol. 2004;151 Suppl 3:U25-37.

30. Glinoer D, Soto MF, Bourdoux P, Lejeune B, Delange F, Lemone M, et al. Pregnancy in Patients with Mild Thyroid Abnormalities: Maternal and Neonatal Repercussions*. J Clin Endocrinol Metab. agosto de 1991;73(2):421-7. 